\title{
Physical Properties of Magnetite Nanoparticles Covered by 11-Mercaptoundecanoic Acid
}

\author{
M. Timko ${ }^{a, *}$, P. KopČAnskÝ ${ }^{a}$, M. Antalik ${ }^{a}$, M. Simsikova ${ }^{b}$, E. VAlusova $^{a}$, \\ M. MOLCAN ${ }^{a}$ AND J. KOVÁC̆ ${ }^{a}$ \\ ${ }^{a}$ Institute of Experimental Physics, SAS, Watsonova 47, 04001 Kosice, Slovakia \\ ${ }^{b}$ Department of Biochemistry, Faculty of Sciences UPJS, Moyzesova 11, 04154 Kosice, Slovakia
}

\begin{abstract}
We have investigated the magnetic behavior of magnetite nanoparticles covered by the 11-mercaptoundecanoic acid around magnetite core prepared by a standard co-precipitation method. The particles show superparamagnetic behavior at room temperature, with transition to a blocked state at blocking temperature $91 \mathrm{~K}$ estimated from zero field cooled and field cooled at 500 Oe experiment. The hysteresis loop measured at $293 \mathrm{~K}$ showed magnetization $32.8 \mathrm{emu} / \mathrm{g}$ at $50 \mathrm{kOe}$ without any coercivity. The mean particle size $(7.1 \mathrm{~nm})$ was determined by fitting a magnetization curve obtained at $295 \mathrm{~K}$ assuming a log-normal size distribution.
\end{abstract}

PACS: 47.65.Cb, 85.70.-w, 75.30.Cr, 75.47.Lx

\section{Introduction}

The biomedical applications of magnetic nanoparticles have experienced a rapid growth due to the benefits in molecular imaging and targeted therapy. Iron oxide nanoparticles are considered as a multifunctional platform, which can be both detected by noninvasive resonance imaging and activated at a distance by a remote magnetic field, allowing controlled manipulation or hyperthermia [1]. Applications in magnetic resonance cell tracking [2], macrophage imaging [3], cancer hyperthermia [4] or tissue engineering [5] are being explored to provide insight into disease mechanism, facilitate diagnostic imaging or design new tools for controlled therapy.

Due to combination of unique magnetic properties, low toxicity and biodegradability [6], the magnetite nanoparticles have great chance to be widely used. Several surfactants have been tested for biocompatibility. Some coatings are degraded enzymatically in vivo [7]. Thus, stability of the resulting particle is of critical importance for mentioned bioapplications. The covalently linked core avoids flocculation of magnetite nanoparticles due to their loss shell [8].

In this contribution we propose the surface coating with 11-mercaptoundecanoic acid around magnetite core prepared by a standard co-precipitation method. The nature of those nanoparticles as well as their innovation of physical properties has been studied. Techniques, such as attenuated total reflection Fourier transform infrared spectroscopy (ATR-FTIR), electrophoretic mobility measurements on Zeta Sizet Nano ZS have been used to characterize new form of magnetite in the powder and/or in the fluidic form.

* corresponding author; e-mail: timko@saske.sk

\section{Experiments}

Preparation of magnetite nanoparticles covered by 11-mercaptoundecanoic acid: iron(II) chloride $\left(\mathrm{FeCl}_{2} \cdot 4 \mathrm{H}_{2} \mathrm{O}\right)-0.054 \mathrm{~g}$ and iron(III) chloride $\left(\mathrm{FeCl}_{3} \cdot 6 \mathrm{H}_{2} \mathrm{O}\right)-0.0198 \mathrm{~g}$ were dissolved in $10 \mathrm{ml}$ distilled water. Under constant stirring was slightly yellow solution warmed up to $80^{\circ} \mathrm{C}$. Then the surface covering was performed by adding dropwise a $2 \mathrm{~mL}$ alkaline solution of $10 \mathrm{mg}$ of 11-mercaptoundecanoic acid to warmed solution stirred at $300 \mathrm{rpm}$. The resulting magnetic black nanoparticles were settled by magnet and washed 3 times with $5 \mathrm{ml}$ of distilled water. In final step, settled moisture magnetic particles were dried and stored at room temperature.

The fluidic form of the above powder was prepared by its dissolving in water and then ultrasonicating the suspension at room temperature for $45 \mathrm{~min}$ until the suspension becomes fluid enough.

Infrared ATR spectra were recorded on a Nicolet 8700 Fourier transform infrared (FTIR) (Thermo Scientific) spectrometer equipped with Smart OMNI-Sampler (diamond crystal), deuterated triglyceride sulfate (DTGS) detector and a $\mathrm{KBr}$ beamsplitter. All spectra were collected for 64 scans at a resolution of $4 \mathrm{~cm}^{-1}$ in range of $4000-400 \mathrm{~cm}^{-1}$ with a $150 \mathrm{~cm}$ aperture. Spectra were referenced to a background spectrum previously recorded on the crystal without the nanoparticles and cleaned under the same conditions as for the covered crystal. The electrophoretic mobility of coated nanoparticles was determined using a Zeta Sizer Nano ZS from Malvern Instruments.

The magnetization properties were measured by SQUID magnetometer of Quantum Design in a magnetic field (up to $50 \mathrm{kOe}$ ) and in a temperature range $2-300 \mathrm{~K}$. 


\section{Experimental results}

\subsection{ATR FT IR spectroscopy}

The ATR FT IR spectra of mercaptoundecanoic acid, magnetite nanoparticles and modified particles by mercapto-undecanoic acid are presented in Fig. $1 a, b$ and $c$. Spectrum of mercaptoundecanoic acid (Fig. 1a) displayed very broad signal at $3094 \mathrm{~cm}^{-1}$ due to the stretching vibration of hydroxo groups and two bands at 2928 and $2847 \mathrm{~cm}^{-1}$ arised to the $\nu_{\text {as }}(\mathrm{C}-\mathrm{H})$ and $\nu_{\mathrm{s}}(\mathrm{C}-\mathrm{H})$ vibrations of $\mathrm{CH}_{2}$ groups. Signals at 1540 and $1402 \mathrm{~cm}^{-1}$ due to symmetric and asymmetric $\nu(\mathrm{C}=\mathrm{O})$ vibrations, which are consequence of ionisation of carboxylic groups into carboxylic anions in alkaline $\mathrm{pH}$.

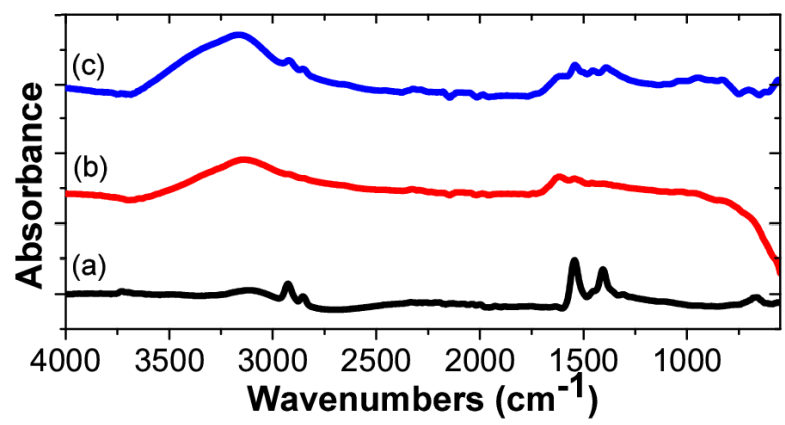

Fig. 1. The FTIR spectra of solid samples: (a) 11-mercaptoundecanoic acid; (b) pure magnetite nanoparticles; $(c)$ mercaptoundecanoic modified particles.

For spectrum of magnetite nanoparticles (Fig. 1b) are characteristic peaks at $3132 \mathrm{~cm}^{-1}$ and $1625 \mathrm{~cm}^{-1}$ which indicates the presence of hydroxo groups on the surface of nanoparticles.

When compared with pure nanoparticles, the spectrum of capped sample contains new peaks at 2918 and $2850 \mathrm{~cm}^{-1}$ corresponding to the stretching vibrations $\nu_{\text {as }}(\mathrm{C}-\mathrm{H})$ and $\nu_{\mathrm{s}}(\mathrm{C}-\mathrm{H})$ of alkane groups and 1540 and $1397 \mathrm{~cm}^{-1}$ which belong to $\nu_{\mathrm{s}}\left(\mathrm{COO}^{-}\right)$and $\nu_{\text {as }}\left(\mathrm{COO}^{-}\right)$ vibrations. These changes confirmed successful surface modification of magnetite nanoparticles via covalent linking with mercaptoundecanoic acid.

\subsection{Measurement of zeta potential of modified magnetite nanoparticles}

The electrophoretic mobility of coated nanoparticles was determined using a Zeta Sizer Nano ZS from Malvern Instruments and transformed into zeta potential using the Smoluchowski equation. For all experiments $1.28 \mathrm{mM}$ solutions of sample at temperature $25^{\circ} \mathrm{C}$ in the water were used. For the titration of the samples to appropriate $\mathrm{pH}$, the concentrated $\mathrm{HCl}$ and aqueous $\mathrm{NaOH}$ were used. The $\mathrm{pH}$ values ( $\mathrm{pH} 2.0-12.0$ ) measured using the SENSOREX glass electrode before and after the data had been recorded.

\subsection{Physical characterization of the surface charge}

Figure 2 shows the change in zeta potential of the magnetite nanoparticles modified by mercaptoundecanoic acid at different $\mathrm{pH}$ values in the range of $2.00-12.00$. The positive zeta potential at $\mathrm{pH} 2(+17 \mathrm{mV})$ was slightly decreased to value $+11 \mathrm{mV}$ for $\mathrm{pH}$. The negative zeta-potential value of nanoparticles was obtained at $\mathrm{pH} 8$ and became practically constant at higher $\mathrm{pH}$ values (pH 8-12). This observation confirmed that the carboxylic acid groups assembled on the nanoparticle surface are completely dissociated around $\mathrm{pH}$ 8. This type of $\mathrm{pH}-$ -ionizable group underwent protonation below the $\mathrm{pH} 7$ due to obtained positive sign of zeta potential. Thus, the isoelectric point (IEP) of sample can assume the $\mathrm{pH}$ value of 7.2 .

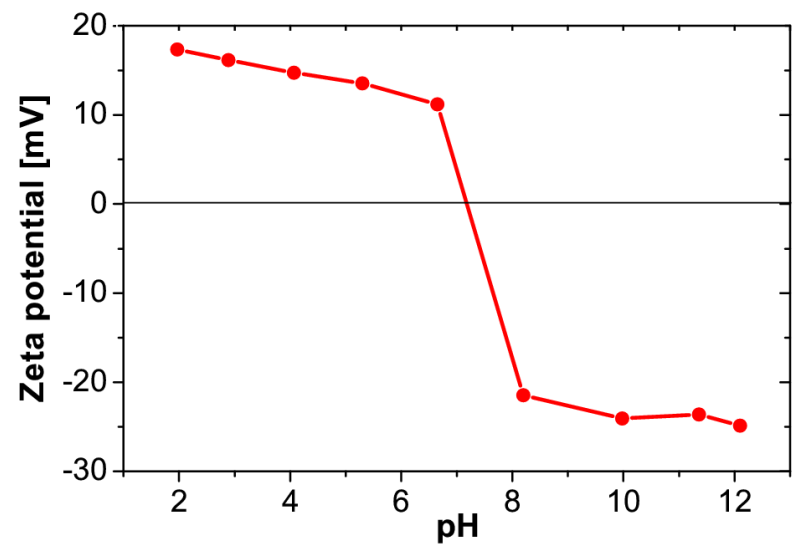

Fig. 2. Zeta potential at different $\mathrm{pH}$ values of coated magnetite nanoparticles by mercaptoundecanoic acid.

\subsection{Magnetic properties}

The magnetization at room temperature and applied magnetic field $50 \mathrm{kOe}$ for prepared sample is $32.8 \mathrm{emu} / \mathrm{g}$. The mean diameter of magnetite core $D=7.1 \mathrm{~nm}$ was estimated from magnetization curve at room temperature (Fig. 3) by the Chantrell method [9]. The hydrodynamic size of nanoparticles (magnetic core + surfactant layer) was estimated to be $10.1 \mathrm{~nm}$. Assuming the core shell structure with a shell of thickness $d$ which does not contribute to the magnetization this smaller value of magnetization can be understood. According to the relation $M_{\mathrm{s}}=92(1-2 d / D)^{3}$ assuming core shell structure with $M_{\mathrm{s}}=92 \mathrm{emu} / \mathrm{g}$ for bulk magnetite and $D=7.1 \mathrm{~nm}$ as a mean particle diameter the value for thickness of shell was obtained as to be $d=1.03 \mathrm{~nm}$. This value of shell thickness is higher but comparable with another values obtained for magnetite nanoparticles covered by oleic acid [10].

The zero field cooled and field cooled curves (Fig. 4) for prepared sample at an applied field 500 Oe indicate the superparamagnetic behaviour with blocking temperature at $91 \mathrm{~K}$. The $\mathrm{ZFC}$ curve exhibits the typical features of an assembly of magnetic particles 


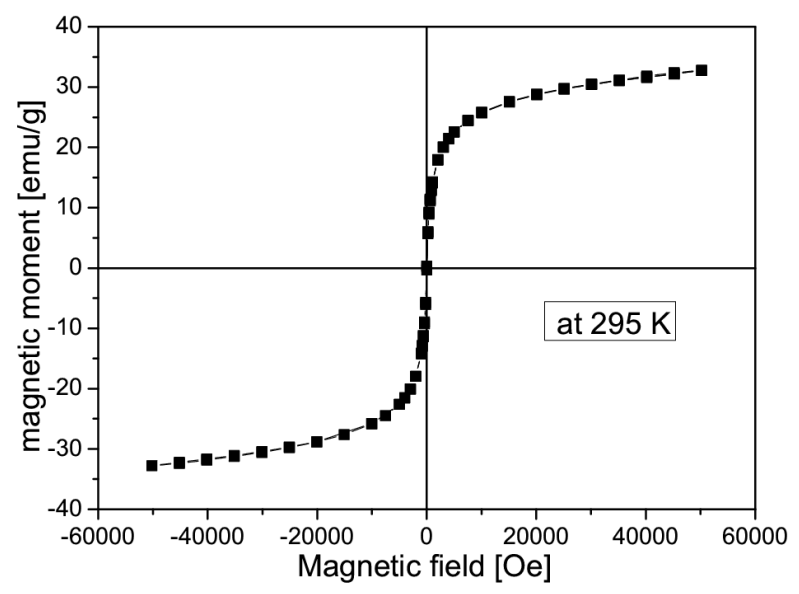

Fig. 3. Hysteresis loop at room temperature.

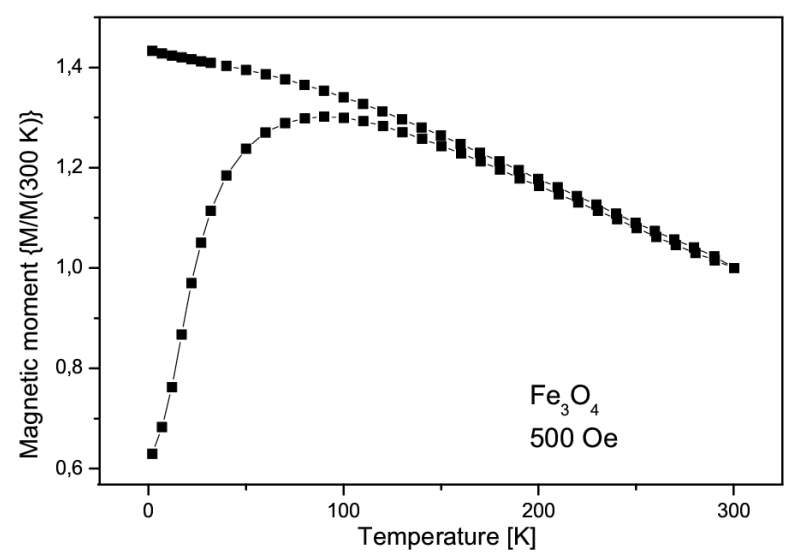

Fig. 4. Zero field cooled and field cooled curves at 500 Oe indicate the superparamagnetic behaviour with blocking temperature $91 \mathrm{~K}$.

with a distribution of blocking temperatures. As the nanoparticles are cooled to very low temperature in the presence of a magnetic field (FC), the magnetization direction of each particle is frozen in the field direction. The ZFC magnetization will exhibit a maximum at the blocking temperature at which the relaxation time equals the time scale of the magnetization measurement.

\section{Conclusion}

In summary, prepared magnetite nanoparticles covered by mercaptoundecanoic acid show typical superparamagnetic properties. Their magnetic behaviors reported here have been interpreted in terms of existence of disordered layer of surface spins, which does not contribute to the magnetization of sample. Further investigations of this disordered shell are now underway using other experimental methods.

\section{Acknowledgments}

This work was supported by Slovak Academy of Sciences (grants VEGA Nos. 2/0077/09, 2/0051/09, 2/0038/09, 1/4290/07 CEX — NANOFLUID), Slovak Research and Development Agency under the contract No. APVV-0171-10 and Development of technology of magnetic fluids for biomedical applications Project No. 26220120021, 26220220005 and 26220120033.

\section{References}

[1] Q.A. Pankhurst, N.K.T. Thanh, S.K. Jones, J. Dobson, J. Phys. Chem. D, Appl. Phys. 22, 224001 (2009).

[2] J.W. Bulte, Am. J. Roentgenol. 193, 314 (2009).

[3] Tang, K.H. Muller, M.J. Graves, Z.Y. Li, S.R. Walsh, V. Young, U. Sadat, S.P.S. Howarth, J.H. Gillard, Atherioscler. Thromb. Vasc. Biol. 29, 1001 (2009).

[4] B. Thiesen, A. Jordan, Int. J. Hyperthermia 24, 467 (2008).

[5] H. Akiyama, A. Ito, Y. Kawabe, M. Kamihira, Biomed. Microdev. 11, 713 (2008).

[6] R. Weissleder, D.D. Stark, B.L. Engelstad, B.R. Bacon, C.C. Compton, D.L. White, P. Jacobs, J. Lewis, Am. J. Roentgenol. 152, 167 (1989).

[7] A. Jordan, P. Wust, R. Scoltz, B. Tesche, H. Fähling, T. Mitrovics, T. Vogl, Int. J. Hyperthermia 15, 705 (1996).

[8] A.B. Bourlinos, A. Bakandritos, V. Georgakilas, V. Tziltzios, D. Petridis, J. Mater. Chem. 41, 5250 (2006).

[9] R.W. Chantrell, J. Popplewell, S.W. Charles, IEEE Trans. Magn. MAG-14, 975 (1978).

[10] P. Dutta, S. Pal, M.S. Seehra, N. Shah, G.P. Huffman, J. Appl. Phys. 105, 07B501 (2009). 